\title{
An e-Learning Platform for SME Manager Upgrade and its Evolution Toward a Distributed Training Environment
}

\author{
Nicola Capuano, Matteo Gaeta, Laura Pappacena \\ CRMPA Centro di Ricerca in Matematica Pura ed Applicata \\ C/O DIIMA - Università degli Studi di Salerno \\ Via Ponte Don Melillo - 84084 Fisciano (SA), Italia \\ Dipartimento di Ingegneria dell'Informazione e Matematica Applicata \\ Università degli Studi di Salerno \\ Via Ponte Don Melillo - 84084 Fisciano (SA), Italia
}

\{capuano,gaeta, pappacena\}@crmpa.unisa.it

\begin{abstract}
The purpose of this paper is to describe the work in progress related to the customisation, the trial and the evaluation of an innovative e-learning platform for manager upgrade in Small and Medium Enterprises (SME) in the framework of an EC funded project named InTraServ and its forthcoming re-engineering process aimed to the adoption of distributed services in the framework of another EC funded project named Diogene. The present e-learning solution includes several state-of-the-art technologies and methodologies such as: metadata and ontologies for knowledge manipulation, fuzzy learner modelling, intelligent course tailoring, case based reasoning, business games and simulation tools. The proposed solution is based on the distribution of working tasks among content provider services, content discovery services, content brokering services, training services, curriculum vitae searching services and collaboration services.
\end{abstract}

Keywords: e-Learning, Web Services, Distributed Environments

\section{INTRODUCTION}

The managerial capacity paradigm argues that a firm's growth is limited by the speed at which it can expand its managerial capacity. This is generally true but it is more and more true for Small and Medium Enterprises (SME) that often don't have a well defined managerial structure causing, in many cases, strategic and decisional lacks that obstruct enterprise's growth.

The learning task for managers is then a critical element for the survival and the success of SME in the global competitive scenery. As quoted in [1] the key challenges to be addressed to satisfy SME training needs concern: distance, time and location (training must serve a dispersed group of learners where and when they require it), flexibility (training can be undertaken between work tasks), availability (training material can be easily located), immediacy (user can obtain quickly solutions to daily working problems).

Starting from these considerations, the EC funded project InTraServ [2] arranged a completely innovative e-learning platform purposed to face and solve these and more issues. The InTraServ project is now approaching the experimentation phase in several SMEs from Italy, UK and Spain.

At the same time, a further EC funded project named Diogene [3] is in progress. Its purpose is to completely re-engineering the architecture of the InTraServ e-learning platform by applying a distributed computing paradigm based on Web Services. This will allow the distribution of training content on different servers, the integration with the forthcoming Semantic Web, the cooperation of different installations of the system, the possibility to create a training offer by combining resources offered by different providers, etc. In this sense it will be a step toward a GRID architecture. 
The paper is organised as follows: the InTraServ project will be briefly described and its main features introduced (section 2). A sketch of the present system architecture will be then presented (section 3) and the new service oriented architecture we have in mind will be introduced (section 4). Some conclusions (section 5) and references will follow.

\section{WHAT'S INTRASERV}

InTraServ [2] is an EC "Trial" project funded under the 5th Framework Programme whose purpose is to customise, try and evaluate an innovative Web-based intelligent e-learning solution for manager upgrade in real SME environments operating in different fields.

Using the InTraServ solution, it will be possible for a manager to take personalised training between working tasks (on-the-job), to evaluate the formal knowledge acquired and to transform it in practical knowledge by experimenting what learnt in simulated situations. When, finally, the manager will master such knowledge, he can apply it inside the organisation. Moreover, using InTraServ, a manager can be supported during his decision making process (just-in-time) by exploiting the CBR-based real case solver component.

The InTraServ e-learning platform is now ready and accessible through the InTraServ portal [2]. It includes four courses (Business Decision, Marketing Management, Marketing Research and Management Control) in three languages (Italian, English and Spanish) and several Business Games (BGs). The following paragraphs describes briefly its innovative features with respect to commercial e-learning platforms presently available on the scene.

\subsection{Metadata and Ontologies for Knowledge Management}

All InTraServ learning material is organised in learning objects (LO) indexed through IMS compliant metadata [4] in order to let the system know what each one of them is about and how it can be used during the learning process.

To provide, also, information about LO relations and interdependency, InTraServ applies ontologies [5] allowing to design abstract, simplified views of training domains. Within InTraServ, ontologies are used to define and relate concepts of a training domain with four kinds of relations: (is_part_of, requires, suggested order and explains) and, also, to link concepts to LOs [6], [7].

\subsection{Fuzzy Learner Modelling}

InTraServ infers and maintains a learner model compliant with the IMS-LIP standard [8] composed by a cognitive state and a set learning preferences. The cognitive state stores, for each concept of a specified training domain, the knowledge degree reached by the learner represented as a set of fuzzy numbers [9] (allowing, in this way, to manage uncertainty in the evaluation process).

Learning preferences, instead, include all information about learner cognitive abilities and perceptive capabilities i.e. to which typology of resources a specified learner is more receptive [6], [7].

\subsection{Intelligent Course Tailoring}

An InTraServ course is composed by an user selected set of learning goals (key concepts that the learner has to learn) and by a learning path (a sequence of learning objects that has to be used to provide, to a specific learner, all necessary knowledge to fully understand chosen goals).

Different learners can require different paths to learn the same goals depending on their learner models. For this reason, InTraServ provides an automatic curriculum generation procedure: the learner can choose what to learn (goals) and let the system organise a personalised learning path for him. Such path can change dynamically during the learning process adapting to learner needs in relation to learner performed activities [6], [7].

\subsection{Case Based Reasoning}


InTraServ gives learners the possibility to solve daily working problems by exploiting a CBR methodology [10] i.e. through a sub-system able to solve new cases comparing the current problem with similar solved problems in a case base and ranking found solutions.

Moreover, the system is able to extend its knowledge by interpreting a new solution in light of similar situations and abstracting out generalisations from experiences. The strength of this approach to problem solving is that the knowledge base is maintained as concrete problem descriptions. In this way the system maintenance could be made by a domain expert rather that by a system expert.

\subsection{Business Games and Simulation Tools}

An important InTraServ feature is the possibility to use a set of advanced simulation tools addressing the business decision process through a "what...if" approach. They refer to a particular learning process phase called "interactive phase": after a learner studied the theory related to a specific topic, he can use such tools to exercise about learned topics.

But a simulation exercise is quite different from a classical exercise: it is based on simulation models that allow concrete experience built up by experimentation and by modification of hypotheses that play a role inside the system under examination [11].

\section{INTRASERV ARCHITECTURE: THE PRESENT}

The main idea behind the InTraServ architecture is that any e-learning application should be supported by a general infrastructure that will put a set of common resources at everyone's disposal and, according to the specific needs of each training domain and application, by a set of further specific resources.

For this reason, the InTraServ architecture, despite that it was customised for the management training domain, is fully adaptable and extensible and can be the basis for the realisation of a virtually infinite set of specific e-learning platforms suitable for any needs, context and domain.

Moreover, the present architecture (see sketch in figure 1), thanks to a strong separation of tasks between different components, will be an optimum starting point to realise a distributed architecture as we will see in the next section.

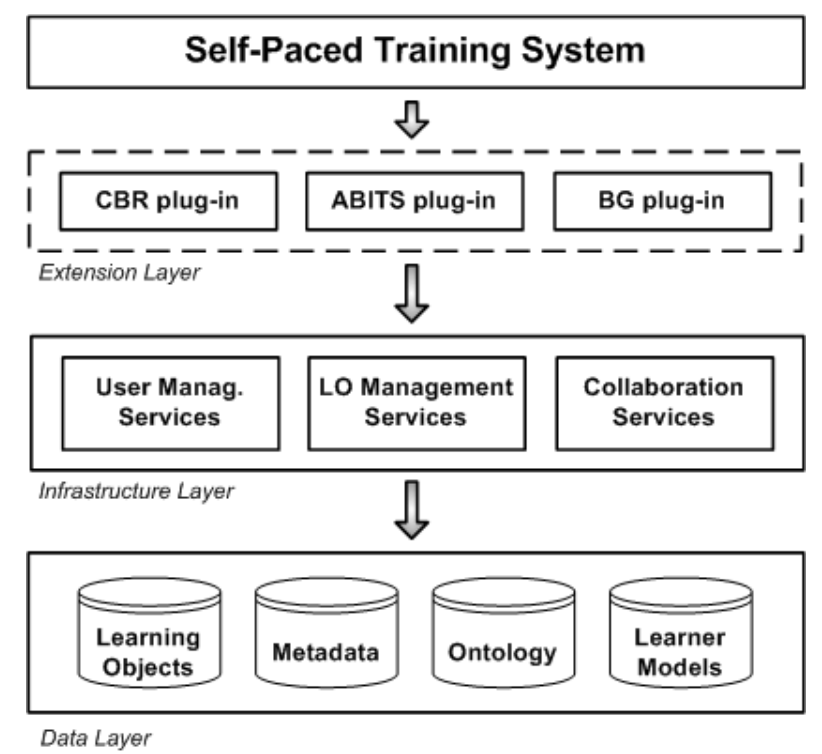

FIGURE 1. The InTraServ Architecture.

As it can be seen, the InTraServ e-learning platform architecture is divided in four layers: Data, Infrastructure, Extension and Self-Paced Training System. 
The Data layer stores all persistent information related to used data structures: Learning Objects, Metadata, Ontologies and Learner Models (see section 2).

The Infrastructure layer, instead, contains all general-purpose e-learning services as described below.

User Management Services: allow registration and logging and provide all user and groups management functions including profiles handling;

Learning Objects Management Services: provide all functions to manage LOs, Metadata and related Ontologies (including access rights handling) and to index and retrieve LOs basing on associated Metadata end Ontologies.

Collaboration Services: include synchronous (text and visual chat) and asynchronous (messaging system and threaded discussion forum) tools for collaboration between users.

The Extension layer, moreover, allows to add at any time new specific domain or context dependent services through the implementation of plug-ins compliant to system specifications. Three plug-ins have been included in the InTraServ Extension layer:

- the Case Based Reasoning (CBR) plug-in that is responsible for case bases reasoning functions (see 2.4);

- the Agent Based Intelligent Tutoring System (ABITS) plug-in that is responsible for "intelligent" training functions (see 2.1, 2.2, 2.3 and [7]);

- the Business Games (BG) plug-in that allows to exploit business games as a particular type of LO (see 2.5).

Finally, the Self-Paced Training System layer includes the front-end related to an instance of the e-learning system (geared toward manager upgrading in SME in the case of the InTraServ project) and allows a filtered access to resources and services provided by the layers below. This layer is obtained via the customisation of a general Web Portal: a dynamic container of panels that give access to underlying services and resources.

The InTraServ e-learning platform has been entirely realised in the Microsoft .NET environment [12], using the ASP.NET language (for the front-end) and the C\# language (for the back-end). Main functions have been wrapped in Web Services in order to allow a standard invocation on the Web from external applications.

\section{DIOGENE ARCHITECTURE: THE FUTURE}

The InTraServ architecture is well structured and suitable for extensions with new services and easily customisable for new training contexts and domains. Nevertheless, it is quite monolithic: it doesn't allow the distribution of training content on different servers, the cooperation of different installations of the system, the possibility to combine training resources offered by different providers, etc.

For this and more reasons, in the framework of further project named Diogene, we completely re-thought the InTraServ architecture in order to allow the distribution of training resources based on Web Services.

Diogene [3] is an EC funded project aimed to design, implement and evaluate with real users an e-learning Web brokering environment for ICT individual training able to support learners during the whole cycle of the training, from the definition of objectives to the assessment of results through the construction of custom courses.

The e-learning system that will be realised under Diogene will use some InTraServ state-of-theart technologies like metadata and ontologies for knowledge manipulation, fuzzy learner modelling, intelligent course tailoring but, also, will include a set of innovative features like dynamic learning strategies, Semantic Web openness, Web services for Learning Object handling and IPR management, Curriculum Vitae generation, maintenance and searching facilities, free-lance teachers support and assisted Learning Objectives definition. 
The architecture we have in mind for Diogene is sketched in figure 2. As it can be seen, it derives, partially from the InTraServ architecture in the distribution of tasks among component/services.

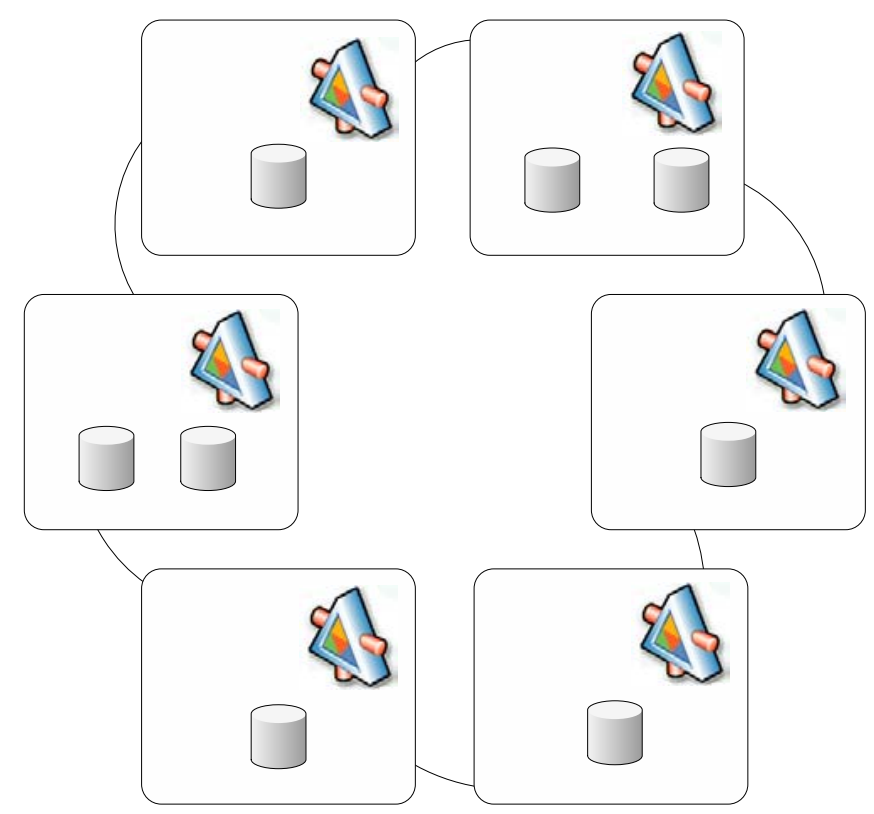

FIGURE 2. The Diogene Architecture.

The meaning of shown Diogene training services is described below.

- Content Provider Services. They are installed in content provider organisations and are used to host training content and provide a standard SOAP-based interface [12] to allow remote access to it. They provide search and retrieval functions on the local repository via metadatabased queries and are able to perform e-commerce transactions between users requesting content and the provider organisation itself to buy the access to content for limited periods of time. They are an extended version of the InTraServ Learning Objects Management Services.

- Content Discovery Services. They are able to extract training content directly from the Web of the present and of the future generation (Semantic Web). Through a keyword-based text categorisation algorithm they are able, where absent, to automatically extract metadata from textual learning objects and to link them to ontology concepts. Through a mixed approach based on keyword and ontologies, moreover, they are able to bypass compatibility problems between different ontological representations of the same domain.

- Content Brokering Services. They are broker of training content. They maintain indexes of learning objects of registered Content Provider and Discovery Services and allow users to use metadata- and ontology-based queries to find the right provider with the right content. They include, also, some course tailoring capabilities (see 2.3) to generate ad-hoc courses by assembling content from registered Content Provider and Discovery Services.

- Training Services. They are responsible for the delivery of courses and for the provision of course management and execution functions. They deal, moreover, with "intelligent" training functions like learner modelling, course tailoring, assisted objective definition and learning strategies upgrading. They don't maintain any local training content but strictly interact with Content Brokering Services or directly with Content Discovery and Provider Services to obtain and combine learning objects. They include and extend the InTraServ Self Paced Training System plus the User management Services and the ABITS plug-in.

- CV Searching Services. They provide search engine capabilities on Learner Models Databases of registered Training Services in order to let third parties interested to hire certified staff to find qualified professional (with respect to privacy requirements). They will maintain, moreover, statistics of received requests in order to rank required competencies. 
- Collaboration Services. They will support social interactions, mentoring and information exchange by providing users a set of collaborative synchronous and asynchronous facilities. They will be able to automatically arrange groups among users of registered Training Services by individuating and grouping learners with similar needs and/or profiles. They are an extension of InTraServ Collaboration Services.

The following figure depicts possible interactions between multiple instances of Diogene services hosted by different organisations. They will constitute the Distributed Training Environment (DTE) of Diogene.

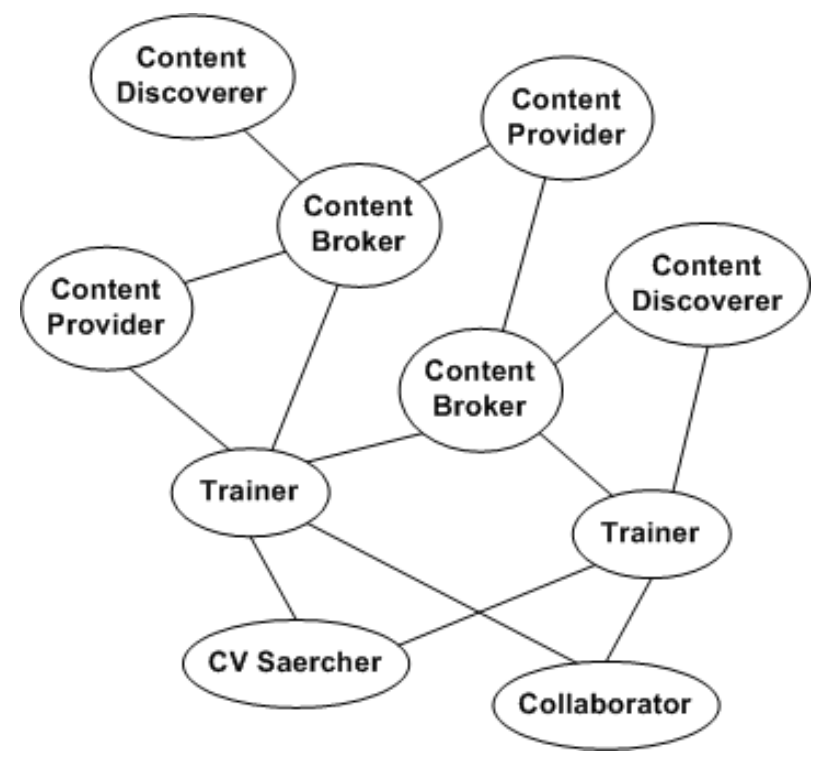

FIGURE 3. Interactions Among Diogene Services.

It's obvious that the distributed architecture designed for Diogene presents several advantages with respect to the InTraServ centralised one.

The maintenance of Learning Objects on content provider servers allows content provider organizations to manage and organize content locally and, in the same time, to overcome property right related problems.

- The networking of different content provider servers filtered through content brokers allows to compose training offer aggregating content from different providers in order to best match user needs.

- The Web is full of training content and the forthcoming Semantic Web paradigm will make it semantically understandable by machines. Content Discovery Services exploit such power to find useful and freeware learning material and to make it available to the DTE.

- The collaboration between peers is of a paramount importance to improve the training experience. Collaboration Services are able to find on the whole DTE (rather then on a single installation of the training service) learners with similar interests and to put them in contact within a co-operative environment.

- Learner models contains useful data about the know-how of students and their acquired competencies: CV Searching Services are able to find qualified professionals all over the DTE and to put them in contact with hiring companies.

\section{CONCLUSIONS}

In this paper we described the e-learning platform realised under the framework of an EC funded project named InTraServ, its innovative features, its architecture and the evolution of such architecture toward a distributed services paradigm (more suitable for a GRID environment) in the context of another EC funded project named Diogene. 
The InTraServ project started in December 2001 and will end in May 2003. The experimentation phase with about 20 managers of Italian, English and Spanish SME is now in course. Apart the InTraServ project, CRMPA decided to experiment the realised e-learning platform also in several Italian academic contexts (like the University of Salerno, of Roma3 and of Molise) in four different courses about computer science and mathematics as a support for teachers in their usual didactic activities. Such kind of experimentation will start in March 2003.

The Diogene project, instead, started in April 2002 and will end in March 2004. The architecture design phase of Diogene is running toward its end and the implementation phase is recently started. A first prototype of the Diogene e-learning platform will be available in September 2003. After the end of Diogene, the next step will be to made the DTE GRID available.

\section{REFERENCES}

[1] European Commission. Trials and Best Practice Addressing Advanced Solutions for On-theJob Training in SMEs. FP5 IST Action Line III.2.3 Background Paper.

http://www.proacte.com.

[2] Web Site "InTraServ: Intelligent Training Service for Management Training in SMEs" FP5 IST Project (IST-2000-29377). http://www.InTraServ.org.

[3] Web Site "Diogene: a Training Web Broker for ICT Professionals" FP5 Project (IST-200133358).

http://www.diogene.org.

[4] IMS Learning Resource Meta-data Specification Version 1.2.2. (2001) Public Draft Specification. IMS Global Learning Consortium.

http://www.imsproject.org/metadata/index.cfm.

[5] Fensel, D. (2001) Ontologies: a Silver Bullet for Knowledge Management and Electronic Commerce. Springer-Verlag.

[6] Capuano, N., De Santo, M., Marsella, M., Molinara, M., Salerno, S. (2001) Personalised Intelligent Training on the Web: A Multi Agent Approach. Electronic Business and Education, Recent Advances in Internet Infrastructures, Kluwer: Multimedia Systems And Applications Series, vol. 20, chap. 5 .

[7] Capuano, N., Marsella, M. Salerno, S. 2000) ABITS: An Agent Based Intelligent Tutoring System for Distance Learning. Proceedings of the International Workshop on Adaptive and Intelligent Web-Based Education Systems. ITS 2000, Montreal, Canada.

[8] IMS Learner Information Package Specification Version 1.0. , (2001) Public Draft Specification. IMS Global Learning Consortium.

http://www.imsproject.org/profiles/index.cfm.

[9] Dubois, D., Prade, H. (1980) Fuzzy Sets and Systems - Theory and Applications. Academic Press.

[10]Kolodner, J., (1993) Case-Based Reasoning. Morgan Kaufmann Publishers.

[11]Albano, G., Miranda, S., SalernoS., (2002) e-Learning e Apprendimento Manageriale. Volume Didamatica 2002 "e-Learning: metodi, strumenti ed esperienze a confronto" - pp. 253-266, 2002.

[12] Macdonald, M. (2003) Microsoft .NET Distributed Applications: Integrating Web Services and Remoting. Microsoft Press. 